\title{
Religion et éthique : de la règle autoritaire à la contextualisation réflexive des normes
}

\author{
Liliane Voyé et Karel Dobbelaere
}

\section{(e) OpenEdition}

Journals

Édition électronique

URL : http://journals.openedition.org/ress/547

DOI : $10.4000 /$ ress. 547

ISSN : 1663-4446

Éditeur

Librairie Droz

Édition imprimée

Date de publication : 1 septembre 2003

Pagination : 151-165

ISBN : 2-600-00878-0

ISSN : 0048-8046

Référence électronique

Liliane Voyé et Karel Dobbelaere, «Religion et éthique : de la règle autoritaire à la contextualisation réflexive des normes ", Revue européenne des sciences sociales [En ligne], XLI-126 | 2003, mis en ligne le 30 novembre 2009, consulté le 19 avril 2019. URL : http://journals.openedition.org/ress/547 ; DOI $10.4000 /$ ress. 547 


\section{RELIGION ET ÉTHIQUE: DE LA RÈGLE AUTORITAIRE À LA CONTEXTUALISATION RÉFLEXIVE DES NORMES}

Durant des siècles, chaque religion s'est présentée comme la détentrice exclusive de la vérité et du sens de la vie. S'adossant à ce monopole auto-conféré, elle prétendait aussi dès lors avoir l'apanage de la définition des règles qui commandaient l'éthique tant sociale que personnelle, dont les principes et les normes d'application se confondaient avec la morale religieuse. Ces règles et normes étaient considérées comme d'autant plus impératives qu'elles se voyaient ainsi dotées d'un caractère transcendant, les faisant échapper à toute velléité de transgression - du moins repérable, car la marge de liberté et d'interprétation inhérente à chacune d'elles a toujours permis écarts, distancements et violations - et à toute tentative de négociation, tant l'altérité radicale et métaphysique de leur genèse les rendait intangibles. Si toutes les religions avaient, au sein du territoire sur lequel elles exerçaient leur suprématie, la même prétention, le christianisme - et, plus particulièrement encore, le catholicisme - entendait, en tant que religion de l'Europe dominante politiquement et économiquement, imposer sa foi et ses règles morales au reste du monde, considéré comme inférieur et donc à civiliser et à convertir - ces deux actions n'en faisant qu'une. C'est d'ailleurs au nom de cette prétention - qui, certes, permettait de sublimer des motivations plus matérielles: conquête de territoires, de ressources diverses et de pouvoir - que fut, durant des siècles, entreprise par l'Occident chrétien la conquête du monde. Que l'on songe, par exemple, aux Croisades qui inaugurèrent pour le monde arabe, à l'époque largement en avance dans tous les domaines scientifiques, médicaux et techniques, «de longs siècles de décadence et d'obscurantisme» qui continuent aujourd'hui à peser sur les relations Occident-Orient et permettent de comprendre, chez ce dernier, l'existence «d'une alternance souvent brutale entre des phases d'occidentalisation forcée et des phases d'intégrisme outrancier, fortement xénophobe» (Maalouf, 1983: 303). Que l'on songe aussi à la conquête des Amériques que, tout en condamnant «la tyrannie et les œuvres infernales des chrétiens », de las Casas lui-même, dans son traité «De l'unique manière d'attirer tous les peuples à la vraie religion», justifie par le souci de convertir les Indiens qu'il décrit comme «très aptes à recevoir notre Sainte Foi Catholique et à conquérir des mœurs vertueuses» (de las Casas, 1992: 50-51). Ce ne sont là que des exemples parmi tant d'autres qui - comme le montre encore une récente encyclique de Jean-Paul II - illustrent la prétention du catholicisme à détenir « la splendeur de la Vérité » et, par-là, les référents obligés de tout jugement moral dont, dit ce texte, «il est impossible de remettre en question le fondement religieux ultime (sur lequel repose) la dépendance de la liberté par rapport à la vérité » (1993: 5457). 
Cependant, malgré la perdurance d'un tel discours, non seulement la religion - et nous parlerons ici plus particulièrement du catholicisme - tend chez nous à perdre son statut de vérité indiscutable mais l'éthique échappe, elle aussi, tant à l'énonciation que celle-ci prétendrait en donner qu'au contrôle auquel elle entendrait la soumettre. L'éthique devient objet de choix et de débat: elle se «spécialise» par champ, devient «situationnelle» et ses référents se concurrencent, produits qu'ils sont par de multiples donneurs de sens, s'affichant ou non comme tels.

Le contexte où se définit cette éthique se transforme lui aussi: progressivement, les savoirs sont de plus en plus mouvants et transitoires; les technologies nouvelles confrontent à des questions inédites et les communications à distance qu'elles permettent se conjuguent à l'accroissement des possibilités de mobilité des personnes pour faire s'entrechoquer au quotidien des cultures diverses, proposant des visions différentes du bien et du mal, du juste et de l'injuste, du vrai et du faux. En outre, dégagé de ses allégeances ascriptives, l'individu se trouve placé au centre du sens et du projet alors qu'il vit dans des situations qui, marquées par l'incertitude - celle de la famille, du travail,... - , appellent de constants réajustements. Fragilisant les convictions antérieures et relativisant les évidences précédentes, cette incertitude, avec l'insécurité qu'elle engendre, confronte ainsi l'individu en tous domaines - y compris éthique - à une exigence quasi permanente de choix qu'il lui semble de moins en moins possible d'opérer à partir de vérités universelles et intemporelles, énoncées en outre en dehors - au-delà - de la mouvance du quotidien.

Dès lors que la modernité a ainsi arraché «la Vérité » et la loi morale aux théologies et aux cosmologies qui l'avaient enfermée dans un ordre éternel et universel supra-humain, se pose en des termes de plus en plus complexes et mouvants la question des fondements et des régulations du lien social. Et le débat ne se situe plus seulement entre, d'une part, vérités et valeurs universellement incontestables et, d'autre part, comme le dit Boudon (1994: 38) «ethnovérités et ethnovaleurs » qui, dépendant de contextes éminemment divers, entendent se voir reconnaître une égale légitimité. Il se déplace un pas plus avant pour interroger le statut de la vérité et des valeurs là où prime l'individu, ses affinités électives et le mot d'ordre «be yourself» qui le mobilise aujourd'hui.

C'est une réflexion autour de ce propos que voudraient ébaucher ces quelques pages. Elles s'articuleront autour d'une série d'exemples montrant la déformalisation et la désubstantialisation pratique qui opèrent actuellement dans divers aspects de la religion catholique et dans différentes règles morales dont celle-ci se voulait et le fondement et le garant. Elles s'achèveront sur ce qui restera un questionnement: comment assurer le lien social dans un contexte qui peut sembler marqué par le relativisme et même par l'idiosyncrasie et qui, au nom de la liberté, rejette toute prétention de vérité et toute velléité d'énoncer d'autorité des règles de comportement et de coexistence.

Au moment même où le Magistère de l'Eglise Catholique réaffirme solennellement la Vérité indiscutable qu'elle énonce et rappelle que la morale découle d'idées données par une instance ultime transcendante, garante de ses certitudes, on voit se multiplier dans les faits toute une série de modes de déformalisation, de désubstantialisation et de contextualisation qui opèrent dans différents domaines. Nous allons tout d'abord montrer comment ces transformations se manifestent 
parmi «les fidèles» en regardant quel sens ils donnent à leurs pratiques et à leurs croyances, comment ils conçoivent le cours de religion et comment ils envisagent les règles morales. Puis nous verrons brièvement comment il arrive parfois que l'institution ecclésiale elle-même tente de sortir du porte-à-faux qui est souvent le sien aujourd'hui.

\section{DE L'INFIDÉLITÉ DES FIDÈLES}

Si le terme de «fidèles » correspondait assez largement à l'attitude que les catholiques avaient jusque il n'y pas si longtemps à l'égard des diverses règles édictées par l'autorité ecclésiale, on ne peut que constater que désormais, ils se distancient largement de celle-ci et ce, sans culpabilité, qu'il s'agisse des croyances, des pratiques, de l'éthique ou encore de ce qu'ils attendent d'un cours de religion.

\section{Des croyances en dérive}

Alors que l'Eglise catholique continue à se présenter comme la seule et unique détentrice de «la Vérité» - ce qui l'amène d'ailleurs parfois à concevoir l'œcuménisme comme un retour des dissidents dans son giron - nombre d'enquêtes récentes montrent clairement que, même les personnes se disant catholiques, ne se rallient pas d'emblée à cette prétention. Très nombreux en effet sont ceux - particulièrement parmi les jeunes - qui disent considérer que «toutes les religions se valent» et que l'appartenance à l'une ou à l'autre dépend simplement du lieu où l'on est né. Ce relativisme religieux ne s'étend pas seulement aux religions non catholiques, chrétiennes et autres; il s'accommode aussi de croyances et de pratiques issues des nouveaux mouvements religieux, notamment du mouvement New Age et des parasciences: astrologie, transmission de pensée, magnétisme,... Boy et Michelat montrent à ce propos que la croyance en ces dernières est particulièrement élevée chez les catholiques qui ne sont pas pratiquants réguliers (de loin majoritaires), les «sans religion » et les - peu nombreux - pratiquants réguliers - disposant, disent ces auteurs, « de systèmes plus cohérents d'explication du monde» qui les préservent davantage de telles croyances. Et ils ajoutent que «la croyance au paranormal va de pair avec toutes les croyances religieuses, en particulier avec la croyance en l'existence de Dieu et en un au-delà de la mort » (Boy et Michelat, 1994: 212).

C'est précisément de ces croyances fondamentales du catholicisme comme de toute religion que nous voudrions à présent dire quelques mots et montrer comment elles se déformalisent et conquièrent une autonomie vagabonde.

Dieu tout d'abord. Le «Catéchisme de l'Eglise Catholique», datant de 1992, reste ferme sur la définition de celui-ci: «Il y a un seul Dieu par nature, par substance et par essence. Il s'est révélé comme l'Unique [...] Dieu a un nom. Il n'est pas une force anonyme. Livrer son nom, s'est se faire connaître aux autres, c'est en quelque sorte se livrer soi-même en se rendant accessible, capable d'être connu plus intimement et d'être appelé personnellement». Les choses sont claires: Dieu est unique et est une personne, même s'il est «au-dessus de tout ce que nous pouvons comprendre ou dire [...]. Et la Trinité est une: un seul Dieu en trois personnes » (Catéchisme de l'Eglise Catholique, 1998: 54-55 et 63). 
Face à la certitude indiscutable d'une telle affirmation, l'image de Dieu se fait, chez «les gens ordinaires», moins univoque et moins certaine, comme le révèlent diverses recherches récentes, tant quantitatives que qualitatives. Outre la progression - même chez les personnes se disant catholiques y compris chez certaines d'entre elles impliquées dans la vie de l'Eglise - de la non-croyance en Dieu et surtout de l'avancée notoire d'une approche dubitative concernant l'existence de celui-ci, le vocabulaire théologique et catéchétique qui entend le décrire - Dieu comme une personne et comme père, roi, juge, ...-fait le plus souvent place à une avancée sensible d'une image de Dieu vu comme correspondant à «ce qu'il y a de bien et de bon dans le monde et dans l'individu». Devenu immanence, Dieu apparaît ainsi comme une façon de symboliser des pans de soi qu'il s'agit de visiter et de promouvoir pour se réaliser pleinement et se dépasser. Il est d'ailleurs frappant de voir que cette définition rejoint pleinement celle de Prigogine lorsqu'il dit: «Je pense que le mot dieu exprime exactement un dépassement de l'homme, son appartenance à quelque chose qui le dépasse» (Prigogine, 1998: 19-20). L'affirmation de la croyance en Dieu qu'expriment $77 \%$ des Européens est donc bien souvent purement nominale et cache des interprétations diverses, éloignées de la doctrine et renvoyant la plupart du temps à une qualité intra-mondaine, collective parfois mais surtout individuelle. Et on ne peut s'empêcher de voir en ceci une proximité «sauvage» avec le bouddhisme qui propose à ses fidèles des moyens devant les aider à faire advenir leur «boddhéité», cette part cachée et spirituelle d'eux-mêmes, qu'ils appellent indifféremment «le divin», «l'être essentiel», ou le «Soi» et dont l'atteinte leur permettra de conquérir l'harmonie avec euxmêmes et avec l'univers et de participer ainsi à une forme d'énergie impersonnelle.

Ainsi le Dieu de nombre de catholiques se voit-il le plus souvent réinterprété loin de la doctrine: il n'est plus une personne mais une force anonyme; il n'est plus le Tout Autre Transcendant mais participe à l'immanence de chacun avec tout ce qu'implique ce double décrochage pour le rapport aux normes et aux règles: il ne s'agit plus ni d'accueillir «le don de la Révélation divine», ni de se soumettre à l'autorité d'une personne - dont la parole est interprétée «authentiquement» en monopole par le seul Magistère de l'Eglise - mais d'utiliser une force, souvent vue comme intérieure, pour se réaliser soi-même dans le sens d'un projet élaboré en propre, individualisé.

Les glissements qui apparaissent dans la croyance en Dieu ne sont pas le seul exemple de croyances en dérive. Il en va aussi de même pour la croyance en la vie après la mort. A l'étonnement des chercheurs, les deux dernières enquêtes européennes sur les valeurs (1990 et 1999) ont fait apparaître un recul sensible de la conception spécifiquement chrétienne de la vie après la mort - la résurrection. En 1999, non seulement il n'y a plus que $43 \%$ des Européens qui disent croire en l'existence de celle-ci, mais près de la moitié d'entre eux (18\%) affirment croire en la réincarnation, celle-ci se voyant en outre généralement réinterprétée librement, loin de sa conception bouddhiste originaire. Des recherches qualitatives ont aussi montré que, pour certains, la réincarnation correspondrait au retour dans une force vitale générale «alimentant» tous les êtres vivants à travers le temps. D'où la tendance croissante à privilégier l'incinération par rapport à l'inhumation - la première étant considérée par nombre de ceux qui optent pour elle comme accélé- 
rant le processus de réintégration dans cette dynamique de vie (Voyé, 1998: 293296).

Ce ne sont là que deux exemples parmi d'autres mais ils nous semblent indiquer clairement la déformalisation qui travaille aujourd'hui les croyances et les éloigne des contenus qu'entendrait y imposer la doctrine. Derrière une éventuelle survivance des termes - Dieu ou la vie après la mort - se cachent des réinterprétations diverses, qui, à partir des réservoirs symboliques des grandes traditions religieuses ou d'autres sources, construisent librement des petits récits croyants marqués tout à la fois par des choix individuels et par la reconnaissance de soi dans l'une ou l'autre des sensibilités affinitaires que l'on retrouve présentes un peu partout.

\section{Des pratiques au sens délitescent}

Les pratiques religieuses, lorsqu'elles subsistent, n'échappent pas, elles non plus, à de profondes transformations, tant quant à leur forme que quant à leur fond. Constatant tout d'abord que, d'une manière générale, les grands rites de passage s'inscrivent désormais bien plus dans la perspective du respect d'une tradition familiale que dans un souci d'allégeance à l'institution ecclésiale (Van Meerbeeck, 2001; Voyé, 1991), nous allons, pour illustrer les recompositions en cours, nous arrêter un moment sur le cas des funérailles religieuses. Si celles-ci restent le rite de loin le plus largement suivi, elles n'en subissent pas moins d'importantes transformations.

Transformations de formes tout d'abord. On l'a dit: de plus en plus de personnes optent pour l'incinération - que certes l'Eglise catholique accepte depuis les années soixante, mais tout en continuant à dire sa préférence pour l'inhumation. Si tel n'est pas toujours le cas, ce changement peut avoir pour incidence de déplacer le lieu de la cérémonie de l'église paroissiale au crématorium régional, ce qui altère doublement la symbolique. Contrairement à l'église qui est un lieu marqué par une seule religion et dont les formes et le décor traduisent matériellement une théologie spécifique, le crématorium est un espace qui se propose comme neutre, puisque destiné à accueillir toutes les religions et aussi l'absence de l'une quelconque de celles-ci. D'autre part, n'existant qu'en nombre relativement limité, le crématorium échappe à la symbolique de la communauté paroissiale, dont l'importance a toujours été soulignée par l'Institution catholique et dont l'église paroissiale était l'expression tangible. (On notera en passant que cette idée de communauté territorialisée est depuis longtemps battue en brèche par les développements de la mobilité qui nous rendent tous plus ou moins nomades). Par ailleurs, qu'il s'agisse de crémation ou d'inhumation, l'organisation de la cérémonie des funérailles suppose quasiment toujours désormais le passage par une entreprise commerciale totalement indépendante de l'Eglise, entreprise qui place les membres de la famille dans une position de «clients » plutôt que de voir en eux des fidèles, et qui fait jouer au prêtre - autrefois grand ordonnateur de la cérémonie - un rôle de service parmi d'autres.

Plus fondamentalement sans doute, le point nodal de la cérémonie se voit aujourd'hui très souvent déplacé de Dieu vers le défunt, de la résurrection à venir vers la vie passée de celui-ci. En particulier parmi les familles de catégorie sociale moyenne et supérieure, surtout de type plus ou moins intellectuel, c'est en effet 
fréquemment autour du défunt et de sa vie que se cristallise la parole, Dieu n étant parfois qu'évoqué brièvement et on pourrait presque dire «accidentellement» lors de paroles rituelles standardisées, prononcées par le prêtre. Celui-ci se voit dans ces cas placé lui-même alors en position marginale, derrière les membres de la famille et d'amis qui «orchestrent» en quelque sorte le déroulement d'une cérémonie truffée d'évocations les plus concrètes possibles de la vie du défunt: ses qualités, ses habitudes et ses manies; ses amours et ses problèmes; ses goûts musicaux, alimentaires ou autres,... Bref, sa personnalité d'autant plus valorisée que peut être mis en exergue le caractère unique de celle-ci, résultat non de la conformité et de la soumission à des règles mais d'une auto-construction difficile, semée d'embûches et toujours en train de se faire. Quant à ce qui se passe après la mort, comme nous l'avons dit, c'est l'attitude probabiliste et dubitative qui l'emporte bien souvent («je ne sais pas s'il existe quelque chose; j'aimerais que tout ne soit pas définitivement fini mais personne n'est jamais venu raconter quelque chose à ce propos»). Et lorsqu'il y a croyance en l'existence d'un «quelque chose », celui-ci s'inscrit souvent, on l'a vu, dans une ligne que l'on pourrait qualifier d'écologique, en ce sens qu'elle tend à voir l'homme comme participant à l'ensemble de la vie et des cycles qui en assurent la durée reproductive.

Une fois encore, on est ici loin des pratiques et des croyances officielles. La possibilité de survie, lorsqu'elle est évoquée, s'insère souvent dans une matérialité terrestre et la cérémonie des funérailles se rabat sur la vie concrète et connue d'une personne particulière plutôt qu'elle ne tente de sublimer la mort par la promesse d'une vie éternelle dans un au-delà problématique. Individualisation et subjectivisation l'emportent donc sur la conformité à l'orthodoxie et à l'orthopraxie, sans que les dispositifs d'autorité puissent d'une quelconque manière imposer leurs régulations.

\section{Des cours de religion en mutation}

Autre domaine affecté: les cours de religion donnés à l'école. Tels que traditionnellement donnés, ceux-ci étaient conçus comme s'adressant à un public catholique, qu'il s'agissait de conforter dans des croyances et des pratiques transmises par la famille. Ce cours se confondait en général avec une démarche catéchétique, c'est-à-dire qu'il s'entendait comme un effort d'éducation à la foi et à la connaissance de «La Vérité» telle que définie par le Magistère. Il se présentait dès lors comme un savoir exhaustif préconstitué, transmis verticalement et constitué de vérités non problématisées à croire de façon inconditionnelle. Certes, ce type d'inculcation n'engendrait automatiquement ni la croyance, ni la pratique, ni la conformité éthique mais il conditionnait d'autant plus la vision du monde que ses contenus apparaissaient largement partagés par un environnement social assez homogène et même par des institutions publiques - Etat, justice,... - héritières de ces mêmes conceptions, au-delà de toute laïcisation et sécularisation.

Depuis la fin des années soixante, les choses ont profondément changé et le cours de religion s'est déformalisé et désubstantialisé. Pour le démontrer, nous nous baserons sur des documents provenant du Secrétariat de l'Enseignement Catholique en Belgique (Segec) et sur deux recherches récentes conduites en Belgique: l'une auprès d'enseignants d'écoles secondaires catholiques (Maton, 1997), l'autre auprès de parents d'élèves de ce même type d'écoles (Polain, 2002). 
Ces trois sources conduisent unanimement au même constat, que l'on peut résumer en quelques points.

Tant dans ce qu'il propose effectivement que dans les attentes exprimées à son égard, ce cours est vu comme devant proposer une ouverture sur la connaissance des grandes traditions religieuses qui, aujourd'hui, se rencontrent et s'entrecroisent sur un territoire longtemps monopole chrétien et d'où les autres religions étaient regardées avec condescendance sinon purement et simplement ignorées car conçues d'emblée comme erronées et mensongères et réservées à des «infidèles », à des mécréants, voire à des païens.

Outre le caractère innovateur de la chose, témoignant déjà d'un changement substantiel de perspective, il est intéressant d'entendre quelle légitimation est donnée à cette ouverture par le Segec lui-même: il s'agit, dit un document émanant de celui-ci, «de fournir des repères en regard desquels chacun peut se situer: on peut s'en rapprocher, s'en emparer, les discuter, s'en écarter, les mettre au centre ou passer à côté». («En avant l'école !», décembre 2001). Deux choses majeures ressortent à notre avis de cette phrase.

Tout d'abord, celle-ci appelle chacun à se situer face à la diversité religieuse. Il n'est donc plus question ni d'enfermer les élèves dans la connaissance d'une seule religion présentée comme seule acceptable et «vraie», ni de présumer a priori une quelconque adhésion à celle-ci. Tout au contraire, il est fait appel au libre choix de chaque élève, appelé à se positionner personnellement, y compris par un éventuel retrait ou rejet. Bien plus, cette confrontation à la diversité religieuse est envisagée par le Segec comme susceptible de faire surgir une identité qui sera d'autant plus vigoureuse que, construite dans l'échange et le débat deuxième point remarquable de cette phrase -, elle sera plus consciente et plus réfléchie. Il n'est donc plus question de «parachuter» des vérités seules et uniques, à ingurgiter telles quelles; il s'agit d'en appeler à la réflexion des élèves et de les engager dans un processus de réflexivité à la lumière de la diversité des informations reçues et des altérités rencontrées. Il n'est plus davantage question de requérir de la part des élèves une attitude de réception passive d'un savoir déjà là et déjà bouclé, présentant toute chose comme d'ores et déjà résolue définitivement; le but est au contraire de les inciter à s'engager pleinement dans la discussion et l'échange en y prenant effectivement la parole et en émettant leur propre point de vue et leurs sentiments et émotions personnels.

On touche ici à un autre changement qui marque lui aussi le cours de religion et qui ressort plus particulièrement de ce que disent et les enseignants et les parents. Loin que ce cours ne tire sa reconnaissance du rendu docile et en quelque sorte distant d'un discours d'autorité s'exprimant dans des termes généraux, plus ou moins théoriques et en tout état de cause, indiscutables, c'est à partir de la résonance qu'il offre à l'actualité que ce cours est considéré comme susceptible de susciter l'intérêt des élèves. Il s'agit, disent enseignants et parents, d'actualiser l'écriture sainte, de montrer en quoi elle peut aider à analyser des situations actuelles, à réfléchir sur celles-ci, à éclairer des événements ou des comportements. Car, disent-ils, ce qui est relaté dans ces textes ne relève pas de l'histoire mais de la symbolique du récit qu'il s'agit d'apprendre aux élèves à décoder avec les outils dont on dispose aujourd'hui - de la psychanalyse à l'informatique.

A côté de cette ouverture sur l'actualité, le cours de religion apparaît aussi aux enseignants et aux parents comme devant être un moment privilégié pour des 
témoignages et des récits d'expériences vécues par les enseignants, les élèves ou encore des tiers. D'une part, disent-ils, cela montre aux enfants que «rien n'est simple», que «tout doit toujours être réfléchi en fonction d'une situation ou d'un contexte particulier». D'autre part, ajoutent-ils, les enfants y sont sensibles parce que «cela manifeste une implication personnelle, des émotions» et montre que chacun rencontre des difficultés, fait des erreurs et a de façon récurrente à se confronter au doute. Ainsi le cours de religion valorisé est-il celui qui laisse surgir la subjectivité et l'émotionnalité, qui appelle l'implication de soi avec ses questionnements et ses incertitudes, avec ses expériences heureuses ou malheureuses. Il s'agit d'y rencontrer la vérité de chacun infiniment incertaine et changeante et non une vérité risquant d'être perçue comme fanatique et arrogante parce que se prétendant immuable, intangible et définitivement exhaustive.

\section{Une éthique situationnelle et fondée dans l'individu}

Outre que la subjectivité et l'individuation touchent les différents aspects du religieux, elles atteignent aussi l'éthique que, jusqu'il y a peu, celui-ci entendait régir et orienter; il détenait de plus de puissants dispositifs d'autorité assurant la mise en conformité comportementale et bénéficiant pour cela de la large correspondance existant en la matière entre règles civiles et règles religieuses, ces dernières étant largement à l'origine des premières.

L'affirmation de la source religieuse révélée de la loi morale donnait à celle-ci un caractère indiscutable et universel et une force de culpabilisation d'une grande efficacité (Delumeau, 1983). Les choses ont aujourd'hui changé et bien des questions morales qui étaient jusque il y a peu présentées et admises comme déjàdepuis-toujours résolues apparaissent désormais comme relevant du registre de l'interrogation individuelle, nourrie par l'expérience quotidienne concrète et par la prise en considération de la spécificité des contextes et situations.

Les résultats de la dernière Enquête Européenne sur les Valeurs sont très illustratifs de ce glissement du lieu de définition de l'éthique. Ainsi, à la question de savoir s'il y a des définitions claires et absolues du bien et du mal ou si l'appréciation dépend toujours des circonstances, $62 \%$ des Européens partagent ce deuxième point de vue et $31 \%$ seulement le premier. (Q21). Ils sont d'ailleurs $88 \%$ à estimer que le développement de l'individu comme tel est une priorité pour le futur (Q57d). Par ailleurs, alors que 75\% d'entre eux disent vouloir privilégier le sens des responsabilités dans l'éducation des enfants (Q49d), ils ne sont que 30\% à entendre insister chez ceux-ci sur l'obéissance (Q49k). D'une façon générale, l'affirmation de l'autonomie de l'individu et la reconnaissance de son libre arbitre l'emportent clairement sur toute idée de soumission à des règles générales, édictées en dehors de lui.

Cette enquête montre également que la majorité des Européens considèrent que c'est l'être humain en tant que tel, usant de sa liberté, de son jugement et de son expérience, qui doit se sentir appelé à prendre ses responsabilités dans nombre de domaines dont les régulations effectives relevaient traditionnellement du religieux, bien souvent en ces matières suivi par la loi civile. C'est notamment le cas de divers aspects concernant la famille: le divorce et l'avortement, par exemple, s'avèrent acceptables aux yeux de beaucoup, dans certaines circonstances et en référence aux caractéristiques des personnes concernées; et l'homo- 
sexualité perd ses anciens stigmates. La bio-éthique se libère, elle aussi, largement des impératifs de l'Eglise, en particulier l'euthanasie dont la légitimité s'accroît dans de très nombreux pays, en particulier les Pays-Bas, la France et la Belgique (Q65).

Il n'est pas sans intérêt de voir que, par contre, la grande majorité des Européens condamnent les «errements sexuels» de personnes mariées. La chose est d'autant plus intéressante à remarquer que, à la question portant sur les conditions d'un mariage réussi, deux éléments viennent largement en tête: la fidélité (82\%) et le respect et l'appréciation mutuelle (83\%) - suivis par la compréhension réciproque $(77,5 \%)$, la discussion entre les partenaires des problèmes éventuels qu'ils rencontrent $(70 \%)$ et la qualité de la vie sexuelle $(60 \%)$. Et cela en même temps que l'on voit le peu d'importance attribuée à l'homogamie religieuse et politique (respectivement $20 \%$ et $8 \%$ ). Tout ceci semble montrer une sensibilité très forte à la confiance existant dans une relation interpersonnelle, alors que les critères plus sociaux paraissent moins décisifs. Ceci est sans doute d'autant plus significatif que cette même recherche montre que lorsque l'on considère l'éthique «sociale », les exigences sont d'autant plus élevées qu'elles sont perçues comme directement garantes du respect d'autres personnes (fumer dans un lieu public, par exemple) alors que la tolérance est relativement élevée dès lors que ce sont des institutions - et particulièrement l'Etat - qui sont en cause (tricher en matière de taxes, par exemple). Tout ceci semble indiquer que lorsqu'il s'agit d'apprécier l'acceptable, voire le normal, c'est la référence à l'individu et à la relation interpersonnelle qui priment alors que les figures d'autorité, Eglise ou Etat, ont perdu leur capacité de faire respecter les règles qu'elles ont édictées. Ceci ne va d'ailleurs pas sans inquiéter l'Eglise, le cardinal belge Danneels, «papabile» notoire, y voyant un effet de «la sensibilité démocratique de notre époque, où l'on détermine soimême les règles et où on peut les changer en fonction des circonstances » (Danneels, 1995: 409)

Ayant perdu leur caractère sacré, «l'idée d'une dépendance radicale de l'être humain à l'égard de certaines normes transcendantes (qui) restaient, même sécularisées, d'essence théologienne» (Ferry, 1996: 87) se dissipe donc pour faire place à des normes éthiques qui renvoient de plus en plus à la conscience de l'individu, à son évaluation personnelle du bien et du mal. Certes cela ne signifie pas qu'il soit pour ce faire sans référence aucune mais il peut désormais s'inspirer de sources diverses - plus ou moins compétentes d'ailleurs! - et construire par luimême, de façon plus ou moins rationnelle, cohérente et sincère son propre jugement moral; ce jugement n'a en outre rien de définitif ni de général mais peut sans cesse être remis en question en fonction tant des circonstances que de l'évolution de la personne elle-même, appelée de façon permanente à se «fabriquer» par un incessant agir réflexif: «nous sommes non pas ce que nous sommes mais ce que nous faisons de nous-mêmes » (Giddens, 1991: 75). C'est en ce sens aussi qu'il faut comprendre l'impératif d'authenticité, si fréquemment évoqué tant à l'égard de soi-même qu'à l'égard des autres.

De sacrée et par-là universelle et intemporelle qu'elle était, l'éthique tend ainsi à devenir situationnelle et individuelle soumise à une contextualisation réflexive, conduisant à sa déformalisation, à sa subjectivisation, et au relativisme et la frappant largement de cette même incertitude qui, nous l'avons vu, corrode les croyances. 


\section{UNE ÉGLISE EN TIMIDE AGGIORNAMENTO?}

Si les «fidèles » prennent ainsi distance vis-à-vis de l'autorité ecclésiale, celleci ne reste pas toujours indifférente ni à cette autonomisation de l'individu qui entend désormais gérer par lui-même ses croyances, ses pratiques et le sens qu'il y donne et auto-construire les fondements de sa morale, ni à sa marginalisation dans la société; force lui est d'accepter qu'elle n'est plus «le baldaquin sacré » dont parlait Berger. Deux exemples vont illustrer cette transformation et les efforts - certes timides - que fait l'Eglise pour redéfinir son rôle et le présenter d'une façon qui soit plus acceptable dans le contexte actuel: celui de l'évolution du droit canonique et celui du discours ad extra utilisé par l'Eglise dans ses «performances ».

\section{Des ruptures dans la sédimentation théologique du droit canonique}

Comme le rappelle Christians, alors que le protestantisme de Luther - lequel, en 1520, a brûlé non seulement la bulle papale le condamnant mais aussi plusieurs recueils de droit canonique - nie «la possibilité d'un droit interne aux Eglises (parce que) la nature proprement spirituelle de celles-ci serait incompatible avec un droit qui n'est qu'une technique temporelle» (Christians, 1998: 5), l'Eglise catholique n'a cessé de rappeler que «l'Eglise fondée par Dieu, dispose de tous les moyens nécessaires à sa fin. Elle constitue une société complète et originaire au moins équivalente à celle des Etats »(Christians, 1998: 6), ce qui l'autorise à définir, comme le font ceux-ci, les règles juridiques applicables à cette société et à développer un discours légaliste et dogmatique inflexible. Si les dernières versions des Codex de droit canonique, qui datent de 1983 et 1991, réaffirment cette conception, il n'en est pas moins apparu, au sein de l'Eglise elle-même, diverses critiques portant sur l'abstraction de ce droit et suggérant « dans une perspective pastorale [...], d'adapter les décisions aux circonstances multiformes et aux exigences variées des cas particuliers, contrairement à l'inflexibilité de la discipline canonique»(Christians, 1998: 8). De telles critiques n'en sont pas restées au niveau du débat puisque, comme le note Christians, on peut repérer des signes concrets de changement, en particulier l'introduction, dans certains diocèses, de la figure de médiation dont l'originalité, par rapport à la situation précédente découlant du droit canonique, est qu'elle «introduit à la fois des éléments de déformalisation normative et des instances de dialogue. Ainsi, par exemple, l'insuffisance des procédures canoniques classiques face au traitement de la pédophilie du clergé a-t-elle conduit, aux Etats-Unis, à l'instauration de commissions canoniques nouvelles, composées non seulement de juges ecclésiastiques mais aussi de laïcs mariés, de psychologues et de parents de victimes antérieures » (Christians, 1998: 19) - la multiplication des points de vue venant ainsi renverser le monopole de l'expertise théologique. Et l'auteur de faire remarquer que si un tel changement intervient au niveau de l'application du droit, il ouvre la voie à un questionnement sur l'élaboration même de la norme, qu'il s'agirait, elle aussi, de contextualiser et de repenser en termes procéduraux.

Si le droit canonique accueille ainsi timidement certaines révisions de sa traditionnelle «sédimentation théologique», plus largement, l'Eglise prend peu à peu 
conscience de la nécessité qui s'impose à elle «d'un constant examen et d'une incessante purification de sa propre vie, de sa législation, de ses institutions, de ses plans d'action» (Paul VI, 1974, cité par Etchegaray dans la «Documentation Catholique», 1990: 322) en référence aux changements que connaît la société et notamment face à l'affirmation pressante des droits de l'homme. (Il n'est pas sans intérêt de remarquer qu'à l'inverse, il est aujourd'hui de moins en moins rare de voir les tribunaux «étatiques » argumenter en tenant compte de normes religieuses qui viennent à leur tour contextualiser le droit «profane» en faisant intervenir des raisonnements d'ordre religieux. Ainsi dans divers cas de «l'affaire du foulard islamique» a-t-on pu voir des juges qui, tout en se réservant la décision finale, faisaient appel à une autorité religieuse islamique chargée de leur donner en la matière son interprétation du Coran ou même des juges qui se référaient à l'autodéfinition de la norme religieuse par la jeune fille concernée.)

On le voit: même réaffirmée vigoureusement, la norme religieuse s'avère dans les faits de moins en moins imperméable aux particularités de son environnement profane (tout comme de son côté la norme étatique se fait de moins en moins indifférente aux spécificités religieuses diverses qu'introduit le caractère devenu multiculturel de la société dépendant de sa juridiction). Ce double processus, que Christians qualifie «d'internormativité» (Christians, 1996) témoigne sans conteste, nous semble-t-il, de la relativisation de l'universalisme proclamé de l'Eglise (de même qu'il questionne - mais ce n'est pas ici notre propos - la séparation de l'Eglise et de l'Etat et plus particulièrement le caractère «laïque» de celui-ci).

Mais le droit canonique n'est pas le seul lieu institutionnel qui montre parfois ainsi un certain souci d'être davantage en prise avec le monde contemporain.

\section{D’une Vérité révélée aux Droits de l'homme}

De manière constante, l'autorité romaine ne cesse de réaffirmer le caractère impératif de ses exigences doctrinales et éthiques. Que l'on songe, pour n'évoquer que quelques exemples récents, à l'encyclique Humanae Vitae s'opposant de façon radicale à tout type d'avortement, à la lettre papale redéfinissant de façon dite irrévocable l'interdiction d'accès des femmes à la prêtrise, à l'instruction concernant la censure et le ré-établissement du «nihil obstat» (1992), ou encore à celle exprimant l'intention - non suivie d'effets - de faire signer aux professeurs des universités catholiques un «serment de loyauté», équivalent à une profession inconditionnelle de foi et d'obéissance à l'Eglise (1990). «Ad intra», l'Eglise catholique s'accroche (Dobbelaere et Voyé, 1991: 219) ainsi à un pouvoir qu'elle n'a plus, tentant d'imposer son autorité disciplinaire et ses règles à une population qui ne les entend pas ou qui y reste indifférente.

C'est un autre discours que tiennent parfois certains acteurs majeurs de l'Eglise lorsqu'ils s'adressent à l'ensemble de la population, qu'elle soit catholique ou non. Ils sortent alors de leur fonction spécifique pour entrer dans un rôle de «performance» au sens où l'entend Luhmann: une institution exerce sa fonction lorsqu'elle intervient dans son champ propre; on parle de performance pour désigner la capacité qu'elle peut avoir d'aider à résoudre certains problèmes ou questions relevant d'autres champs et que ceux-ci s'avèrent impuissants à résoudre par eux-mêmes (Luhmann, 1990). En ce qui concerne l'Eglise, il arrive 
régulièrement qu'elle se propose ainsi d'elle-même comme ressource, en particulier pour le champ politique, ou même qu'elle soit interpellée par celui-ci à de telles fins. Un exemple intéressant concerne l'Europe.

De sa propre initiative mais aussi à la requête de leaders majeurs de celle-ci (en particulier Delors), l'Eglise se présente parfois comme susceptible sinon chargée de changer l'image de cette Europe. Accusée d'être d'abord sinon exclusivement une vaste machine économique, celle-ci serait «en quête d'une âme». La capacité de l'Eglise de jouer un rôle majeur dans la réalisation d'un tel projet est fréquemment exprimée. Ainsi le pape insiste-t-il régulièrement sur la spécificité de la culture européenne et des valeurs qui y sont attachées (et dont, dit-il, une des racines est chrétienne). Lorsqu'il parle en dehors de son champ - à des diplomates ou à des leaders politiques du monde ou lors de ses visites à l'ONU et au Parlement européen - le pape, et d'ailleurs plusieurs personnages majeurs de son entourage, définissent bien souvent ces valeurs en termes de «droits de l'homme». La chose est piquante ! En effet, non seulement il est alors question de droits et non plus de devoirs - ce qui éloigne d'une morale d'interdits et de prescrits - mais surtout on ne peut oublier que la hiérarchie catholique s'est violemment opposée à la Déclaration universelle des droits de l'homme de 1789 parce qu'elle l'associait à la Révolution française et à la République laïque (Luneau et Ladrière, 1989: 172-178). Mais ce qui importe particulièrement pour notre présent propos, c'est que cet appel aux Droits de l'homme dédogmatise le discours en recourant à une référence et à un langage élaborés loin de l'Eglise et plutôt associés à son effacement; c'est aussi qu'il situe l'objectif non plus dans l'atteinte d'une vie surnaturelle extra terrestre en particulier à travers sacrifices et contraintes, mais bien dans la réalisation dans ce monde d'une vie qu'il s'agit « de rendre plus heureuse» en profitant pour cela des opportunités nouvelles existantes. Et la contribution de l'Eglise à cette tâche est précisée: il ne s'agit pas pour elle de s'engager dans la définition de moyens pratiques et concrets mais simplement de mettre à la disposition des hommes et des gouvernants son «expertise en humanité » (Voyé, 1997: 170).

Ainsi, lorsqu'ils entendent s'adresser «au monde», les leaders catholiques abandonnent-ils souvent tout langage disciplinaire, au contenu rigide et impératif, pour s'ouvrir à des paroles non marquées du sceau théologique - ce qui conduit dès lors à s'interroger sur la spécificité de l'éthique chrétienne et sur ce que la foi «ajoute encore à la morale laïque commune» car «le respect de la personne humaine, le souci de l'autre, de sa dignité ou de sa souffrance ne sont plus des principes dont le christianisme aurait le monopole. Pour beaucoup même, ils apparaissent à bien des égards comme construits contre lui» (Ferry, 1996: 57). C'est donc ici aussi à une déformalisation du discours de l'Eglise que l'on assiste, avec l'abandon de toute référence expresse à l'origine transcendante des règles morales, avec aussi la reconnaissance de l'homme individuel en tant que porteur de droits et non plus comme soumis aux exigences d'une vérité révélée, issue d'une extériorité radicale. Ce faisant, la porte est ouverte au changement et à l'interprétation qu'excluait cette origine transcendante. 


\section{CONCLUSION}

Tout au long de ces pages associant ces transformations à la sécularisation (Dobbelaere, 2002) et à la montée de l'affirmation de l'individu comme lieu du sens et du projet, nous avons parlé de déformalisation, d'individualisation, de subjectivisation, de dédogmatisation,... de divers aspects du champ religieux et du domaine éthique qui en a été longtemps directement dépendant. Et nous avons vu que, même si elle cherche parfois à s'arroger le monopole du discours vrai - et ainsi à asseoir son pouvoir (Berthelot, 1996b: 181) - l'Eglise catholique est de moins en moins suivie dans cette voie et est elle-même amenée à s'ouvrir un tant soit peu aux mutations contemporaines.

Les illustrations que nous avons apportées de ces changements permettentelles pour autant de parler de relativisme au sens où l'entend Berthelot: «un énoncé n'a d'autre validité que celle, toujours singulière, de ses effets pragmatiques dans la situation où il s'insère »; «toute connaissance est fonction de son contexte d'énonciation» (Berthelot, 1996a: 48 et 88)? Certes, Berthelot parle des sciences mais son propos est stimulant pour analyser ce qui se passe aujourd'hui dans les domaines religieux et éthique. Même si, dans ces cas, on ne se situe pas dans le registre de la recherche d'une objectivité universelle et intemporelle (contrairement à ce que voudrait l'autorité ecclésiale), ce champ ne peut davantage fonctionner sur «le principe du tout est bon» (Boudon et Clavelin, 1994: 38). Là comme ailleurs, il s'agit donc, comme y insiste Busino, de «dépasser le dualisme désastreux de l'objectivité universellement admise et de la subjectivité incommunicable, de la réalité qui s'impose à tous et des valeurs individuelles et arbitraires, des vérités nécessaires et éternelles et des savoirs soumis aux contingences sociales et historiques, d'une rationalité techno-scientifique et d'un décisionnisme existentiel réduisant les choix à des décisions irrationnelles et à des actes de foi » (Busino, 1994: 48).

Certains indices montrent en fait que, dans le champ qui nous occupe ici, l'alternative est bien souvent dépassée dans les faits. Si l'Eglise n'a plus la capacité de contraindre, d'imposer ses croyances, ses pratiques et ses règles éthiques, on ne tombe pas pour autant dans un relativisme échevelé, ouvert à une réflexivité qui serait totalement individuelle. Bien au contraire, on assiste à l'émergence non d'une fragmentation radicale mais de «petits systèmes croyants » (Hervieu-Léger, 2001-88) et de logiques éthiques consistantes, qui nous semblent manifester un sens partagé sinon par tous, du moins par beaucoup, ce partage de sens étant source de validation et de légitimité. Ainsi, dans le domaine religieux, si c'est la posture dubitative qui l'emporte, elle s'accompagne de diverses régularités de vision et d'attente: celle de la reconnaissance des dimensions émotionnelles et corporelles de l'homme; celle de la quête de la réalisation plénière de soi «ici bas », de l'épanouissement personnel tant physique qu'affectif et spirituel - ces différentes dimensions étant vues non comme dissociées mais bien comme complémentaires et interreliées (d'où le succès de divers types de soins, techniques et thérapies insistant sur la globalité de leurs effets); celle encore de l'importance accordée à la vie dans ce monde qui s'apprécie de façon auto-référentielle, trouvant son sens en elle-même. De mêmes régularités surgissent en matière éthique, qui tournent toutes autour du respect de la personne, de son droit à la dignité et au bien-être, à l'intégrité corporelle et à l'autodétermination. Que 
l'on songe à l'avortement, largement admis lorsque la vie de la mère est en danger ou lorsque l'enfant risque de naître fortement handicapé mais rejeté lorsqu'il s'agit d'une forme de contraception. Que l'on pense aussi à l'euthanasie, de plus en plus acceptée lorsque la personne est réduite à un état végétatif, lorsque ses souffrances lui semblent intolérables - mais ce, pour autant qu'elle en ait fait ellemême la demande.

Au-delà des questions particulières qui viennent d'être évoquées, c'est tous les champs qui sont concernés par de telles transformations. Celles-ci ne vont pas sans interroger le mode de fonctionnement général de nos sociétés ni sans affecter les modalités de tissage d'un lien social qui s'est émancipé de ses anciennes tutelles institutionnelles et des normes impératives qu'elles édictaient. Le cas des champs religieux et éthique semble indiquer qu'à la prétention universaliste antérieure - avec ce qu'elle avait d'ethnocentrique - ne succède pas un relativisme radical, absolutisant la différence et multipliant des «particularismes - ghettos » (Eberhard, 1999: 275). L'effondrement d'une attitude de réception disciplinée et passive de normes stables et exhaustives, supposées définies par une instance ultime fait place non pas à une idiosyncrasie mais à l'expression d'un sens partagé, s'articulant autour de quelques thèmes majeurs. Ainsi si les normes sont devenues négociables en référence à une historicité entendue comme discours situé, elles n'en semblent pas pour autant erratiques. Elles se construisent à partir d'une mobilisation cognitive qui s'enracine dans la «compétence pratique» des acteurs, avec ce que cette proximité apporte d'affectif et d'implication personnelle. Elles visent non plus à construire de la conformité mais à faire surgir une capacité de s'affirmer comme acteur pensant et agissant, avec d'autres plus ou moins différents mais reconnus comme potentiellement porteurs aux aussi de cette capacité.

Université catholique de Louvain

et

Katholieke Universiteit Leuven

\section{RÉFÉRENCES BIBLIOGRAPHIQUES}

Berthelot, Jean-Michel (1996a), Les vertus de l'incertitude. Le travail de l'analyse dans les Sciences Sociales, Paris, PUF;

- (1996b), «La science est-elle soluble dans le social?», in Revue Européenne des Sciences Sociales/Cahiers Vilfredo Pareto, vol. XXXIV, $\mathrm{n}^{\circ} 104$, pp. 181-186.

Boudon, Raymond et Clavelin, Maurice (1994), Le relativisme est-il résistible? Regards sur la sociologie des Sciences, Paris, PUF.

Boy, Daniel et Michelat, Guy (1994), «Premiers résultas de l'enquête sur les croyances aux parasciences » in: Colloque de la Villette, La pensée scientifique et les parasciences, Paris, A. Michel.

Busino, Giovanni (1994), «Réponse à Raymond Boudon» in: Le relativisme est-il résistible? Regards sur la sociologie des sciences, Boudon, R. et Clavecin, M., pp. 44-49.

Christians, Louis-Léon (1996), «Approches juridiques de la normativité religieuse», Conférence à l'UCL, Louvain-la-Neuve, Une procéduralisation des droits religieux?, Louvain-la-Neuve, Carnets du Centre de Philosophie du Droit, UCL.

Danneels, Gotfried (1995), «Pauvre Eglise!» in: La Documentation catholique. 
Danze, Marie-Annick (2001), «De la mort à la vie. Analyse sociologique des rites funéraires et des représentations de la mort», Mémoire UCL.

De Las Casas, Bartolomé (1992), Très brève relation de la destruction des Indes, Paris, La Découverte.

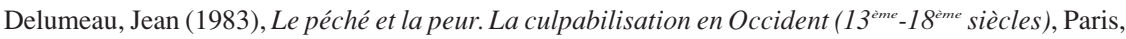
Ed. Fayard.

Dembour, Marie-Bénédicte (2001), «Following the Movement of a Pendulum: between Universalism and Relativism » in: J. K. Cowan, M. B. Dembour, R. A. Wilson, Culture and rights. Anthropological Perspectives, Cambridge, Cambridge University Press, pp. 56-79.

Desplanque, Thibault (1997), Cendres de Belgique. Analyse sociologique de la crémation en Belgique, Mémoire UCL.

Dobbelaere, Karel (2002), Secularization: An Analysis at Three Levels, Bruxelles, P.I.E. Peter Lang.

Dobbelaere, Karel et Voyé, Liliane (1991), «Western European Catholicism since Vatican II» in: H. R. Ebaugh, Religion and the Social Order, vol. 2: Vatican II and U. S. Catholicism, Greenwich/London, Jai Press Inc., pp. 205-231.

Eberhard, Christophe (1999), «Pluralisme et dialogisme. Les droits de l'homme dans une mondialisation qui ne soit pas seulement une occidentalisation.» in: La Revue du MAUSS, n ${ }^{\circ}$, pp. 261-279.

Erikson, Thomas E. (2001), «Between Universalism and Relativism: a Critique of the UNESCO Concept of Culture» in: J. K. Cowan, M. B. Dembour, R. A. Wilson, Culture and rights. Anthropological Perspectives, Cambridge, Cambridge University Press, pp. 127-148.

Ferry, Luc (1996), L' homme-dieu ou le sens de la vie, Paris, Grasset.

Giddens, Anthony (1991), Modernity and Self-Identity. Self and Society in the Late Modern Age, Cambridge, Polity Press.

Halman, Loek (2001), The European Values Study: A Third Wave (Source book of the 1999/2000 EVS Surveys), WORC, Tilburg University.

Hatch, Elevin (1997), «The Good Side of Relativism », Journal of Anthropological Research, vol. 53, nr 3, pp. 371-381.

Hervieu-Leger, Danièle (2001), «Crise de l'universel et planétarisation culturelle: les paradoxes de la 'mondialisation' religieuse» in: Bastian, Champion, Rousselet, La globalisation du religieux, Paris, L'Harmattan, pp. 87-96.

Jean-Paul II (1993), La splendeur de la Vérité, Paris, Mame/Plon.

Luhmann, Niklas (1990), Essays on self-reference, New York, Columbia University Press.

Luneau, René et Ladriere, Paul (1989), Le rêve de Compostelle: vers la restauration d'une Europe chrétienne? Paris, Ed. Centurion.

Maalouf, Amin (1983), Les croisades vues par les Arabes. La barbarie franque en Terre Sainte, Paris, J.-Cl. Lattès.

Maton, Christine (1997), A propos de la spécificité de l’enseignement catholique, Louvain-la-Neuve, Rapport de stage UCL.

Polain, Céline (2002), Le cours de religion : vers une logique de la construction plutôt que de l'imposition, Mémoire UCL.

Prigogine, Ilya (1998), De l' être au devenir, Liège, Ed. Alice.

Van Meerbeeck, Anne (2001), Het doopsel : een familie-ritueel [Le baptême: un rituel familial], Thèse de doctorat, KULeuven.

Voyé, Liliane (1991), «Les jeunes et le mariage religieux » in: Social Compass, vol. 39, n²4, pp. 405416;

- «Religion in Modern Europe» in: Inoue, Globalization and Indigenous Culture, Institute for Japanese Culture and Classics, Tokyo, pp. 154-186;

- (1998), «Death and Christmas revisited. Beyond Secularization: search for meaning. » In: R. Laermans, B. Wilson, J. Billiet, Eds. Secularization and Social Integration, Leuven, Leuven University Press. 